\title{
Channel Prediction and Predictive Vector Quantization Aided Channel Impulse Response Feedback for SDMA Downlink Preprocessing
}

\author{
Du Yang, Wei Liu, Lie-Liang Yang and Lajos Hanzo \\ School of ECS, University of Southampton, SO17 1BJ, UK \\ Tel: +44-23-8059 3364; Fax: +44-23-8059 4508 \\ E-mail: dy05r,wl03r,lly,lh@ecs.soton.ac.uk; http://www-mobile.ecs.soton.ac.uk
}

\begin{abstract}
Invoking SDMA in the Down-Link (DL) has the potential of increasing the achievable throughput with the aid of linear transmit preprocessing, provided that the Channel Impulse Responses (CIRs) of all users and all antenna elements are known at the DL transmitter. However, in a Frequency Division Duplex (FDD) system, since these CIRs have to be transmitted by the Mobile Terminals (MTs) to the Base Station (BS), they are naturally out-dated. Hence, we proposed a periodical CIR update scheme employing a channel predictor at the DL transmitter for predicting the CIR taps for each future symbol transmission instant and hence to mitigate the performance degradation imposed by the associated signalling delays. Moreover, a Predictive Vector Quantizer (PVQ) is used at the MTs for compressing the CIRs before their uplink transmission. Compared to a conventional Vector Quantizer (VQ), PVQ has significantly reduced the CIR feedback bit rate. Hence, with the aid of the same feedback bit rate, the new PVQ scheme can provide more accurate CIR information or support a channel having a higher Doppler frequency.

Index Terms-SDMA, channel prediction, predictive vector quantiza-
\end{abstract} tion, feedback, out-dated

\section{INTRODUCTION}

Spatial Division Multiple Access (SDMA) Downlink (DL) System has been widely investigated in recent years [1]-[3]. The principle of SDMA is that multi-user communication can be supported within the same time-slot and same bandwidth by exploiting the users' different spatial signatures, i.e. Channel Impulse Responses (CIRs). When the cooperation of different users has to be avoided due to complexity and security reasons, transmit preprocessing at the BS constitutes an attractive means of Multi-User Interference (MUI) mitigation. Hence, by employing both multiple antennas and transmit preprocessing at the BS, the DL systems' throughput may be increased without any extra cost in terms of power, bandwidth or Mobile Terminal (MT) complexity.

However, all the known transmit preprocessing algorithms designed for SDMA applications require accurate knowledge of the CIRs of all antennas of all users. There are basically two CIR acquisition methods [4]. One of them employs pilot symbols transmitted from the MTs in the Up-Link (UL) and assumes that the DL CIRs are similar to the CIRs in the UL, which is an often-used assumption in Time Division Duplex (TDD) systems [5]. The other technique relies on using a feedback link from the MTs transmitted through a bandwidth-limited UL control channel, which is applicable for both TDD and Frequency Division Duplex (FDD) system [5]. Nevertheless, both of them are subjected to signal processing, control protocol-induced and propagation delays. Due to the time-variant

${ }^{1}$ The work reported in this paper has formed part of the Core 4 Research Programme of the Virtual Center of Excellence in Mobile and Personal Communications, Mobile VCE, www.mobilevce.com, whose funding support, including that of EPSRC, is gratefully acknowledged. Fully detailed technical reports on this research are available to Industrial Members of Mobile VCE. nature of the wireless channel, neither of them is capable of providing instantaneous CIR information, which results in a performance degradation. Additionally, the feedback imposes an UL throughput loss.

Naturally, channel prediction techniques can be used to mitigate the effect of signalling delay [6]. In this contribution, we considered this problem in a CIR feedback mode, which is the most generally applicable technique. We proposed a periodical CIR update scheme employing a channel predictor at the BS, which mitigates the effects of UL signalling and control protocol-induced delays. Furthermore, we employed a Predictive Vector Quantizer (PVQ), which exploits both the spatial- and time-domain correlation in order to significantly reduce the feedback bit rate compared to the conventional Vector Quantizer (VQ).

In Section II we provide an overview of both the DL and feedback link structure. The channel prediction principles are outlined in Section III, while our quantizer design is detailed in Section IV. Finally, our performance results and discussions are offered in Section $\mathrm{V}$, followed by our conclusions.

\section{System Model}

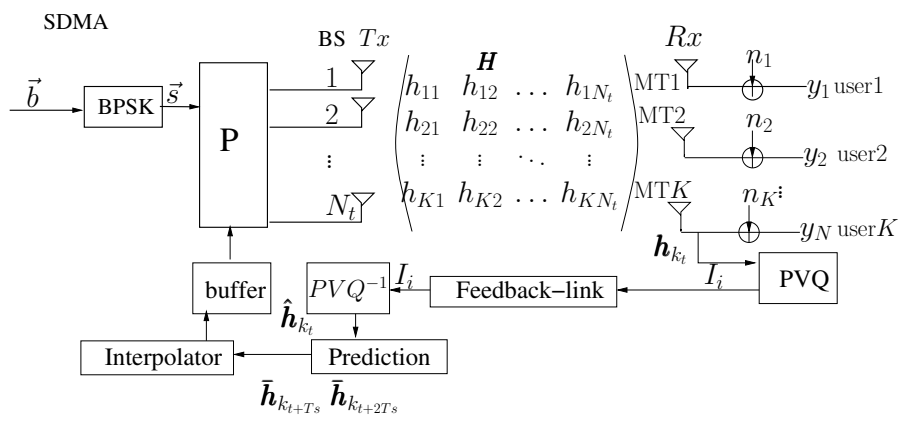

Fig. 1. The System Model of Downlink and Feedback Link

\section{A. Downlink}

Let us consider a Multiple Input Single Output (MISO) multi-user system employing a total of $N_{t}$ DL transmit antennas and a single receive antenna at each MT, as shown in Fig. 1. With the aid of transmit preprocessing, the system's throughput may be increased by a factor of $K\left(K \leq N_{t}\right)$. It can be shown that the length $K$ vector $\boldsymbol{y}$ received by the $K$ MTs' antennas can be expressed as

$$
\boldsymbol{y}=H P x+n,
$$

where $\boldsymbol{x}=\left[x_{1}, x_{2}, \ldots, x_{K}\right]^{T}$ contains the $K$ users' transmitted symbols. It is assumed that $E\left[\left|x_{k}\right|^{2}\right]=1$ and $x_{k}$ is an independently 
and identically distributed (i.i.d) uniform random variable. In (1) $n$ is the length $K$ noise vector, hosting complex-valued random Gaussian variables having a zero mean and a variance of $\sigma^{2} / 2=1 / 2 \mathrm{SNR}$ per dimension, where SNR represents the signal-to-noise ratio averaged over all the $N_{t}$ transmission channels. Furthermore, in (1) the matrix $H$ containing the CIRs between the transmit and receiver antennas can be expressed as

$$
\begin{aligned}
\boldsymbol{H} & =\left[\boldsymbol{h}_{1}^{T}, \boldsymbol{h}_{2}^{T}, \ldots, \boldsymbol{h}_{K}^{T}\right]^{T}, \\
\boldsymbol{h}_{k} & =\left[h_{k 1}, h_{k 2}, \ldots, h_{k N_{t}}\right], k=1,2, \ldots, K,
\end{aligned}
$$

where $\boldsymbol{h}_{k}$ represents the $k$ th user's CIRs, also often referred to as the spatial signature corresponding to the $k$ th user detecting $x_{k}$. In this contribution, we assumed that the propagation environment exhibits rich scattering and the channels are spatially independent time-variant flat Rayleigh fading media. Each element of $\boldsymbol{H}$ is represented as an identical independent complex Gaussian variable with zero mean and unit variance. Futhermore, $\boldsymbol{P}$ is the $\left(N_{t} \times K\right)$ element transmitter preprocessing matrix, which is given by

$$
\begin{array}{r}
\boldsymbol{P}=\left[\boldsymbol{p}_{1}, \boldsymbol{p}_{2}, \ldots, \boldsymbol{p}_{K}\right]=\mathrm{f}(\boldsymbol{H}), \\
\text { s.t. } \operatorname{Tr}\left(\boldsymbol{P} \boldsymbol{P}^{H}\right) \leq K,
\end{array}
$$

where $\boldsymbol{p}_{k}$ models the preprocessing techniques to be invoked for mitigating the interference among the DL signals of the $K$ users $x_{k}, k=1, \ldots, K$. Furthermore, $\boldsymbol{P}$ is designed as a function of $\boldsymbol{H}$ and subject to power constraints. The design of $\boldsymbol{P}$ has been widely studied [7]-[9]. In this contribution Transmit Minimum Mean-square Error (TxMMSE) preprocessing schemes are considered for the sake of having a low-complexity implementation. The algorithm is detailed in Appendix VI-A.

\section{B. Feedback Link}

To provide the CIRs for the BS, the MTs' CIRs $\boldsymbol{h}_{k_{t}}\left(N_{t} \times 1\right)$ have to be sampled and estimated every $T_{s}$ seconds and then forwarded to the quantizer for digitisation. Since a non-dispersive channel is assumed, each of the $N_{t}$ number of CIRs is represented by a single complex-valued tap. Instead of the quantized CIR $\hat{\boldsymbol{h}}_{k_{t}}$ itself, the quantized CIR prediction error $\hat{\boldsymbol{e}}_{k_{t}}$ is represented as a PVQ codeword at the index $I_{i}$, which is fed back to the BS. The PVQ codebook is designed off-line and assumed to be known by both the MTs and BSs. As a result, the BS reconstructs the CIR tap $\hat{\boldsymbol{h}}_{k_{t}}$ by inverse quantization. The most recent $p$ number of reconstructed CIR taps are fed into the channel predictor to predict the future CIRs taps. Considering that the BS encounters a delay of $\tau$ (usually $\tau<T_{s}$ ), two future CIR taps corresponding to two consecutive pilots should be predicted in advance, which are $\overline{\boldsymbol{h}}_{k_{t+T_{s}}}, \overline{\boldsymbol{h}}_{k_{t+2 T_{s}}}$ . Then an interpolator is invoked to reconstruct the CIR taps for each transmitted symbol among $\overline{\boldsymbol{h}}_{k_{t}}, \overline{\boldsymbol{h}}_{k_{t+T_{s}}}$ and $\overline{\boldsymbol{h}}_{k_{t+2 T_{s}}}$. The predicted and interpolated CIR taps will be restored and periodically updated in the BS's DL transmit buffer. This process, as seen in Fig. 1, provides the predicted CIRs, which are closer to the actual CIRs than in the absence of prediction.

Both the channel predictor and quantizer design constitute key design issues. They determine the accuracy of both the predicted CIRs and the feedback bit rate, which will be discussed in the rest of this paper. To simplify our discussions, we have assumed perfect channel interpolation and an uncontaminated UL CIR feedback link.

\section{Channel PRedictor}

It is well recognised that the time-variant nature of a narrow band fading channel is characterised by its maximum Doppler frequency $f_{d m}$, which also determines the correlations of the CIR taps as modelled by the Auto-Regressive (AR) process of:

$$
h_{n}=\sum_{k=1}^{p} a_{k} h_{n-k}+w_{n},
$$

where $h_{n}$ is the complex-valued non-dispersive CIR tap at time instant $t=n T_{s}$, and $p$ is the number of previously reconstructed taps fed into the CIR predictor. This correlation property enables us to predict the future CIRs, given the knowledge of the past reconstructed CIRs and the AR process coefficients $a_{k}$. The design of $a_{k}$ is based on the autocorrelation function of the sampled CIR taps, which is known to be given by the zero-order Bessel function of the first kind:

$$
r(\tau)=J_{0}\left(2 \pi f_{d m} \tau T_{s}\right), \tau=1,2, \ldots,
$$

where $T_{s}\left(f_{s}=\frac{1}{T_{s}}\right)$ is the sampling interval. It is plausible that we can predict a vector of consecutive reconstructed CIR taps given their time domain correlation. The linear MMSE predictor's design is given in Appendix VI-B.

Naturally, the accuracy of the prediction can be improved in conjunction with larger values of $p$ and $f_{s}$, but $f_{s}$ should be as low as possible, in order to reduce the feedback bit rate. In our study, we have fixed the value of $p$, and investigated the suitable choice of $f_{s}$.

Futhermore, in our system the past quantized CIR taps were obtained with the aid of the UL. Naturally, the accuracy of the quantizer also affects the accuracy of the prediction. In fact, the predictor is quite sensitive to it. As a result, a high-resolution quantizer constituted by the smallest possible codebook is desired.

\section{QUANTIZER}

\section{A. Conventional Vector Quantizer}

It has been shown in [10] that to achieve the same resolution, a VQ always requires a lower number of bits than a scalar quantizer (SQ). Hence VQs designed for CIR quantization have been intensively studied [4], [11]-[13]. A common technique is to quantize the CIR envelope and phase separately, since their statistical distributions are different, namely Rayleigh and uniform, respectively. The Generalized Lloyd Algorithm (GLA) constitutes the most common codebook generation technique [10]. The cost function used for codebook design is usually the overall Mean Squared Quantization Error (MSQE) between the real CIRs and the quantized CIRs.

However, in our study, the channels are assumed to be spatially independent. In this case, the advantage of using VQs is quite modest. As a result, it is necessary to design a more efficient quantizer to achieve an increased resolution using a low number of bits. In our study, we have used such a conventional VQ as the benchmarker of our PVQ.

\section{B. Predictive Vector Quantizer}

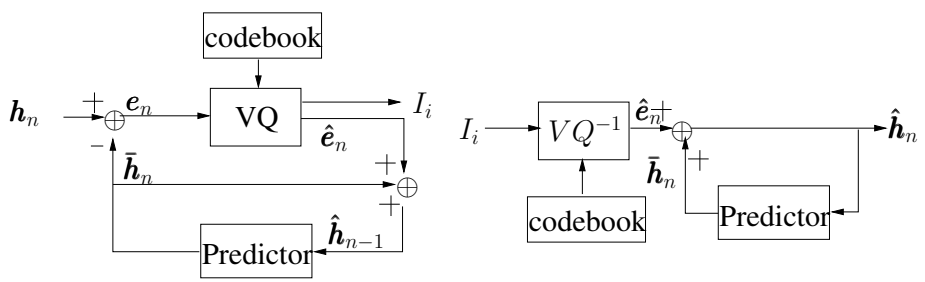

Fig. 2. Structure of the Predictive Vector Quantizer 
Principle of $P V Q$ : Although the CIR taps of the individual antenna elements are typically assumed to be independent of each other, their consecutive time-domain samples are correlated. In order to exploit this time domain correlation, a PVQ may be employed.

Fig. 2 shows the structure of a PVQ. Although in theory the channel predictor order $p$ in (4) and the PVQ's predictor order in Fig. 2 can be chosen to be different, in our design both sere fixed to $p$. Hence the previous $p$ number of quantized CIR taps $\left(\hat{\boldsymbol{h}}_{n-1}, \hat{\boldsymbol{h}}_{n-2}, \ldots, \hat{\boldsymbol{h}}_{n-p}\right)$ are used at the MTs to predict the current estimated CIR tap. The predicted value $\overline{\boldsymbol{h}}_{n}$ is subtracted from the current estimated value $\boldsymbol{h}_{n}$ and the resultant prediction error $\boldsymbol{e}_{n}$ is quantized. The quantization result $\hat{\boldsymbol{e}}_{n}$ is then added to $\overline{\boldsymbol{h}}_{n}$ to generate $\hat{\boldsymbol{h}}_{n}$ for future prediction. The reason of using quantized CIRs instead of the unquantized CIRs at the MTs for channel prediction is to avoid using different predictor inputs at the BS and MTs, since at the BS only the knowledge of quantized CIRs are available. At the same time, the index $I_{i}$ of the codebook representing $\hat{\boldsymbol{e}}_{n}$ is fed back to the BS. At the BS, as seen in Fig. 2, the PVQ decoder first converts the received codebook index $I_{i}$ to the corresponding codeword. Simultaneously, an identical predictor invokes the previously reconstructed CIR taps to predict the current CIR taps. The predicted result is then added to the quantized prediction error in order to reconstuct the current CIR tap.

The PVQ outperforms the conventional VQ, since it quantizes the CIR tap prediction error instead of using a conventional VQ to quantize the channel's envelope or phase. It is clear that the former has a reduced input signal's range. Based on the theorem stated in [10], the overall reproduction error $E\left(\left\|\boldsymbol{h}_{n}-\hat{\boldsymbol{h}}_{n}\right\|^{2}\right)$ is equal to $E\left(\left\|\boldsymbol{e}_{n}-\hat{\boldsymbol{e}}_{n}\right\|^{2}\right)$. Hence, given a certain feedbak rate, the CIR quantization error will be significantly reduced by the PVQ.

Off-line PVQ Design: As a first step, the predictor and the quantizer are designed separately, which is referred to here as an open-loop design. The predictor design has already been described in Section III. Since initially no quantizer is available, only the unquantized rather than the quantized CIR samples can be used for prediction and for generating the prediction error. The GLA is employed for the open-loop VQ codebook generation, and the cost function employed is

$$
\mathcal{C}=\arg \min E\left(\left\|\boldsymbol{e}_{n}-\hat{\boldsymbol{e}}_{n}\right\|^{2}\right),
$$

where $\boldsymbol{e}_{n}$ and $\hat{\boldsymbol{e}}_{n}$ are complex-valued vectors and $\mathcal{C}$ represents the complex-valued codebook entry minimizing the quantization error. Once the initial codebook becomes available from the open-loop design, we can create a joint GLA-aided closed-loop predictor and quantizer design, where we use the quantized CIRs for prediction and generate a new prediction error sequence for the new closedloop codebook design. After several such design iterations, an improved codebook will be generated with the aid of the closedloop design. When using a large PVQ codebook, the closed-loop design provides only modest improvements, but in case of a less accurate quantizer, closed-loop design can substantially improve the accuracy of the PVQ. This is illustrated in Table I, in terms of the quantization error variance versus the number of design iterations using $f_{s}=10 f_{d m}, N=5$ and $N=10$ bits, respectively. It shows that for $N=5$ bits, the quantization error decreases by employing the closed-loop design, but almost no improvement is obtained when using $N=10$ bits.

\section{Simulation Results and Discussion}

The system parameters used in our simulations are summarized in Table II. The important assumptions are as follows:

\begin{tabular}{|l|l|l|}
\hline & $E_{m m s e}$ for $N=5$ & $E_{m m s e}$ for $N=10$ \\
\hline Open-loop design & 0.3128 & $5.15 \times 10^{-5}$ \\
\hline 1st iteration & 0.0786 & $5.2 \times 10^{-5}$ \\
\hline 4th iteration & 0.0406 & $5.18 \times 10^{-5}$ \\
\hline
\end{tabular}

TABLE I

COMPARISON OF THE QUANTIZATION ERROR VARIANCE FOR DIFFERENT NUMBER OF DESIGN ITERATIONS, WHEN USING $f_{s}=10 f_{d m}, N=5$ AND $N=10$ BITS

\begin{tabular}{|l|l|}
\hline Number of BS antennas & 2 \\
\hline Number of MT antennas & 1 \\
\hline Number of users & 2 \\
\hline Channel code & $R=\frac{1}{2}$, conv code [15 17] \\
\hline Modulation scheme & BPSK \\
\hline Transmit preprocessing scheme & TxMMSE \\
\hline Predictor type & Linear MMSE \\
\hline Predictor order & $p=2$ \\
\hline Normalized Doppler Frequency & 0.01 \\
\hline \multicolumn{2}{|c|}{ TABLE II } \\
\hline \multicolumn{2}{|c|}{ SYSTEM PARAMETERS }
\end{tabular}

1) The channels are assumed to be spatially independent flat Rayleigh fading with a maximum Doppler frequency of $f_{d m}$;

2) The interpolation between the predicted CIR taps is assumed to be perfect sinc-interpolation in order to focus our attention on the quantizer and predictor design;

3) The UL feedback link is assumed to be uncontaminated;

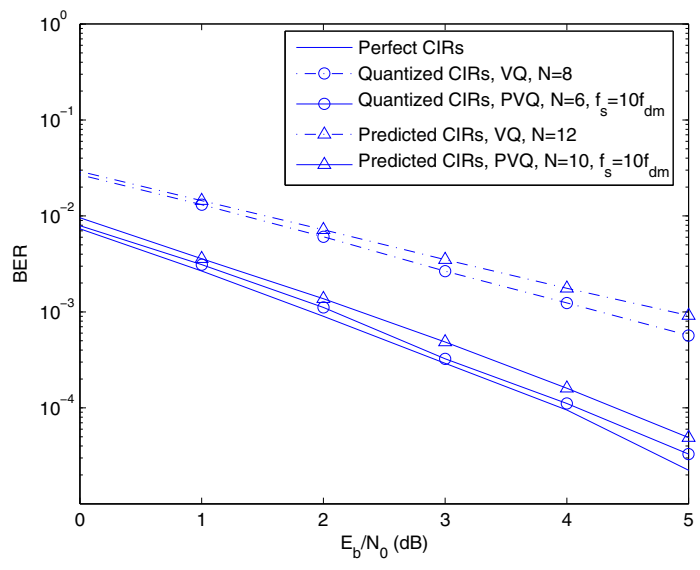

Fig. 3. BER versus $E_{b} / N_{0}$ with perfect, quantized and predicted CIRs using PVQ or VQ, respectively, assuming perfect DL channel estimation.

Fig. 3 illustrates the attainable Bit Error Ratio (BER) versus $E_{b} / N_{0}$ performance. In this example the DL channel estimation is assumed to be perfect and five specific scenarios were investigated concerning the knowledge of the CIR used for transmit preprocessing.

1) The first scenario assumes that the transmitter employs perfect CIR knowledge for preprocessing;

2) The second investigation assumes finite-precision but instantaneous CIR knowledge, which is provided by a PVQ $(N=$ $\left.6, f_{s}=10 f_{d m}\right)$;

3) The third scenario is similar to the second one, but a VQ using 8 bits is employed instead; 


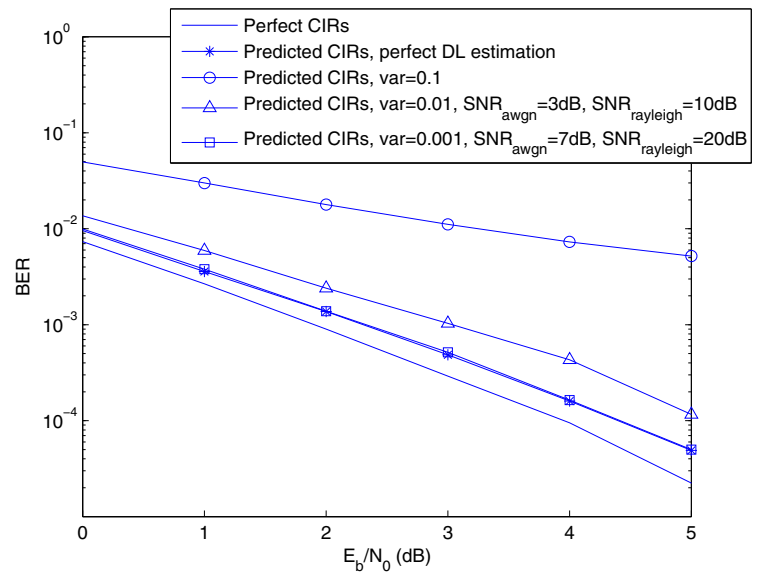

Fig. 4. BER versus $E_{b} / N_{0}$ with perfect and predicted CIRs using PVQ, respectively, assuming imperfect DL channel estimation.

4) The fourth case assumed predicted CIR knowledge using a PVQ $\left(N=6, f_{s}=10 f_{d m}\right)$;

5) The last one also investigated the attainable performance using predicted CIR knowledge, but provided by a $12 \mathrm{bit} \mathrm{VQ}$.

The simulation results of Fig. 3 indicated that

1) The BER performance achieved over a wide SNR range obtained by using perfect CIR knowledge is almost achievable by employing a channel predictor and a PVQ;

2) The performance of using perfect CIRs is closely approximated with the aid of 10-bit quantization employing PVQ, while 20 bits are required for the conventional VQ scheme. Hence, PVQ is more efficient.

To be more practical, Fig.4 illustrates the attainable BER versus $E_{b} / N_{0}$ performance under the assumption of imperfect DL channel estimation. The DL CIR estimation error is modelled as additive complex-valued Gaussian noise. Fig. 4 shows that if the estimation error variance is lower than 0.001 , there is almost no performance loss. This indicates that the DL SNR should be higher than $7 \mathrm{~dB} / 20 \mathrm{~dB}$ for AWGN/Rayleigh channels assuming that the DL pilots used for CIR estimation are BPSK modulated.

In addition to the attainable BER, another important concern is the feedback bit rate expressed as:

$$
R_{f b}=k f_{d m} N, \quad k \in \mathcal{R}^{+},
$$

which may also be referred to as the feedback overhead. In (7), $k f_{d m}$ denotes the feedback information update rate, where $k$ is a positive real number representing the over-sampling factor. If the value of $f_{d m}$ can be estimated and the feedback update rate may be adjusted to match the Doppler frequency, our scheme is capable of reducing the feedback bit rate by employing a more efficient PVQ. For example, for $f_{d m}=30 H z, f_{s}=10 f_{d m}$ and 10 bits per complex CIR tap, the feedback overhead of our scheme becomes $R=10 \times 30 \times$ $10=3 \mathrm{Kbit} / \mathrm{s}$, while the conventional $\mathrm{VQ}$ scheme requires about $R=10 \times 30 \times 20=6 \mathrm{Kbit} / \mathrm{s}$ for achieving a similar performance.

However, in practice, the feedback information is often transmitted through a dedicated control channel, which has a certain maximum allowable information rate. For example, the 3GPP WCDMA-FDD system has 10bits per $0.667 \mathrm{~ms}$ time slot for UL control information signalling including the uplink-pilots, feedback information etc. In this case, our PVQ scheme outperforms the conventional VQ in terms of providing more accurate CIR information and supporting a channel exhibiting a higher Doppler frequency. To simply illustrate this, we assumed that the entire control channel is used for signalling the CIR feedback information. Hence the feedback data rate is $\frac{10 b i t}{0.667 \mathrm{~ms}}=$ $15 \mathrm{Kbit} / \mathrm{s}$. With the aid of our new PVQ scheme, the maximum supported Doppler frequency becomes $\frac{15000}{(10 \times 10)}=150 \mathrm{~Hz}$, while the conventional scheme can only support $f_{d m}=\frac{15000}{(10 \times 20)}=75 \mathrm{~Hz}$.

As a futher example, we considered the scenario when the maximum affordable feedback rate is fixed (e.g. WCDMA-FDD), and the Doppler frequency $f_{d m}$ is known. Then we choose the value of $k$ and $f_{d m}$ in order to achieve the highest-accuracy CIR representation we can, so as to achieve a low BER.

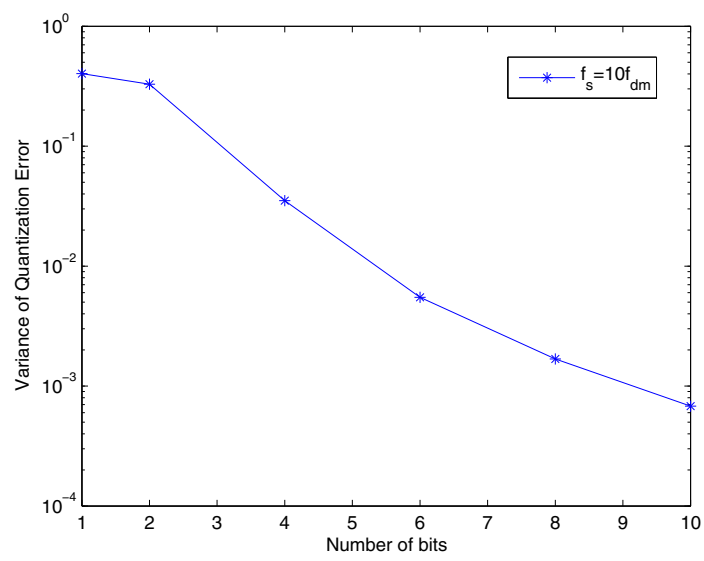

Fig. 5. Quantization error variance of the PVQ versus number of quantization bits when using a sampling rate of $10 f_{d m}$

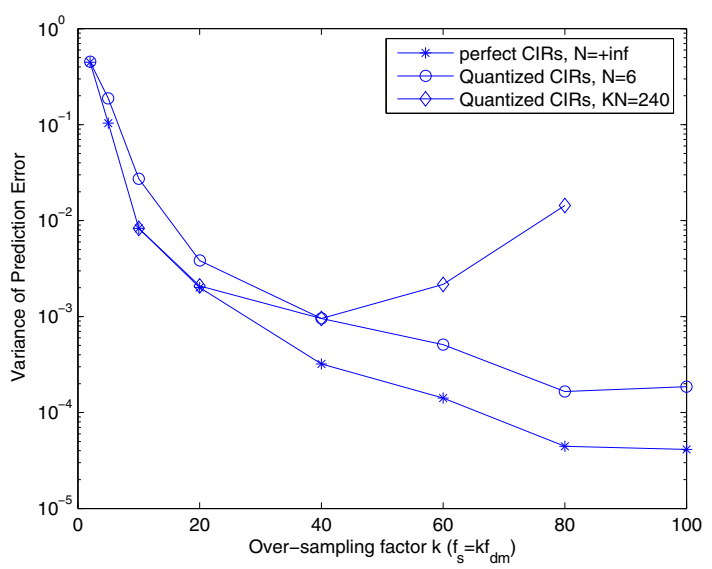

Fig. 6. Prediction error variance versus sampling rate $f_{s}=k f_{d m}$ employing both perfect CIRs and quantized CIRs using $N=6$ bit PVQ as well as a fixed value of $k N b i t, f_{s}=10 f_{d m}$

Fig. 5 shows that the quantization error variance of the PVQ decays near-linearly with the number of quantization bits used for a fixed sampling rate of $f_{s}=10 f_{d m}$. Fig. 6 shows that the prediction error using both perfect CIRs and $N=6$ bit quantized CIRs rapidly decreases upon increasing the sampling rate of $f_{s}=k f_{d m}$. Naturally, a higher sampling rate is preferred. However, the gap between the curves corresponding to perfect CIRs and quantized CIRs widens, 
as the sampling rate increases. Hence a PVQ using a fixed number of codebook address bits improves the achievable performance more significantly at a high sampling rate than at a low sampling rate. To illustrate this phenomenon, we investigated a fixed CIR sampling-rate value of $k f_{d m}=240$ in conjunction with $k=10,20,40,60,80$, respectively. The simulation results of Fig 6 show that as the oversampling factor increases, the prediction error variance first decreases then increases again and has its minimum at $k=40, N=6$. In conclusion, if the value of $k N$ is fixed, the highest prediction accuracy is achieved at a moderate value of $k$ and $N$.

\section{CONCLUSION}

Employing a channel predictor combined with a PVQ to provide predicted CIR knowledge for the transmitter preprocessing arrangement is capable of achieving a BER performance close to that attained with the aid of perfect CIR knowledge across a wide SNR range. The feedback rate required by the PVQ compared to the conventional VQ is dramatically reduced. Hence, at the same feedback bit rate, more accurate CIRs can be provided by the PVQ and a high-Dopplerfrequency channel can be supported. As a result, the DL system's throughput was increased by the PVQ-aided the SDMA scheme, since DL SDMA is capable of supporting more than one user.

\section{APPENDIX}

\section{A. MMSE Transmit Preprocessing}

Provided that the CIR matrix $\boldsymbol{H}$ and the noise variance $\sigma^{2}$ are known at the BS, the design cost function is:

$$
\boldsymbol{P}_{m m s e}=\arg \min E\left(\|\boldsymbol{y}-\boldsymbol{s}\|^{2}\right) \quad \text { s.t.E }\left(\|\boldsymbol{P} \boldsymbol{s}\|^{2}\right) \leq K \text {. }
$$

We can find necessary conditions for $\boldsymbol{P}$ by constructing the Lagrangian function

$$
L(\boldsymbol{P}, \lambda)=E\left(\|\boldsymbol{y}-\boldsymbol{s}\|^{2}\right)+\lambda\left(E\left(\|\boldsymbol{P} \boldsymbol{s}\|^{2}-K\right), \lambda \in \mathcal{R}\right.
$$

and setting its derivatives to $\frac{\partial L}{\partial \boldsymbol{P}}=\mathbf{0}$. By carrying out this derivation [7], the matrix $\boldsymbol{P}$ can be calculated by first calculating:

$$
\boldsymbol{F}=\boldsymbol{H}^{*}\left(\boldsymbol{H}^{T} \boldsymbol{H}^{*}+\sigma^{2} \boldsymbol{I}_{K}\right)^{-1} .
$$

In order to keep the transmit power constant, calculate the power normalization factor as:

$$
\beta=\sqrt{\frac{K}{\operatorname{Tr}\left(\boldsymbol{F} \boldsymbol{F}^{H}\right)}},
$$

where $\operatorname{Tr}(\boldsymbol{X})$ represents the trace of $\boldsymbol{X}$. The MMSE transmit preprocessing algorithm is given by:

$$
\boldsymbol{P}=\boldsymbol{F} \beta \text {. }
$$

\section{B. AR Predictor Coefficients}

Scalar: Provided that $f_{d m}, p, T_{s}$ are known,

1) Calculate

$$
r(\tau)=J_{0}\left(2 \pi f_{d m} \tau T_{s}\right), \tau=0,1, \ldots, p
$$

2) Construct the autocorrelation matrix of the channel:

$$
\boldsymbol{R}=\left(\begin{array}{cccc}
r(0) & r(1) & \ldots & r(p-1) \\
r(1) & r(0) & \ldots & r(p-2) \\
\vdots & \vdots & \ddots & \vdots \\
r(p-1) & r(p-2) & \ldots & r(0)
\end{array}\right)
$$

3) Construct the autocorrelation vector of the channel

$$
\boldsymbol{r}=\left(\begin{array}{c}
r(1) \\
r(2) \\
\vdots \\
r(p)
\end{array}\right)
$$

4) Calcualte the AR predictor coefficient vector $\boldsymbol{a}_{\boldsymbol{k}}$

$$
\boldsymbol{a}_{k}=[a(1), a(2), \ldots, a(p)]^{T}=\boldsymbol{R}^{-\mathbf{1}} \boldsymbol{r} .
$$

$A R$ Predictor Coefficient Matrix: Provided that $f_{d m}, p, T_{s}$ are known, the AR coefficient matrix $\boldsymbol{A}_{k}, k=1,2, \ldots p$ can be determined. The CIR vector $\boldsymbol{h}_{n}$ sampled at time instants $t=n T_{s}$ and having $N_{t}$ non-zero elements can be predicted according to:

$$
\boldsymbol{h}_{n}=-\sum_{k=1}^{p} \boldsymbol{A}_{k} \boldsymbol{h}_{n-k},
$$

where each $\boldsymbol{A}_{k}$ is a $N_{t} \times N_{t}$ matrix.

1) Calculate

$$
r(\tau)=J_{0}\left(2 \pi f_{d m} \tau T_{s}\right), \tau=0,1, \ldots, p
$$

2) Construct

$$
\boldsymbol{R}_{i j}=E\left[\boldsymbol{h}_{n-i} \boldsymbol{h}_{n-j}^{T}\right]=r(|i-j|) \boldsymbol{I}_{N_{t}} ;
$$

3) $A_{k}$ can be calculated based on the equation:

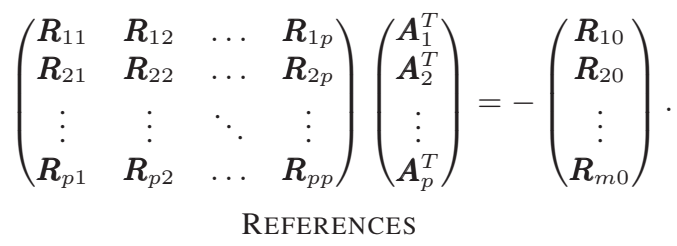

\section{REFERENCES}

[1] C. Farsakh and J.A. Nossek, "Spatial covariance based downlink beamforming in an SDMA mobile radio system", IEEE Transactions on Communications, vol. 46 , pp. 1497-1506, Nov. 1998.

[2] Q.H. Spencer, A.L. Swindlehurst and M. Haardt, "Zero-forcing methods for downlink spatial multiplexing in multiuser MIMO channels", IEEE Transactions on Signal Processing, vol. 52, pp. 2700-2712, August 2005.

[3] C.Y. Wei, J. Akhtman and L. Hanzo, "Iterative Near-Maximum-Likelihood Detection in Rank-Deficient Downlink SDMA System", IEEE Transactions on Vehicular Technology, vol. 57, pp. 653-657, Jan. 2008.

[4] D.J. Love, R.W. Heath, W. Santipach and M. Honig, "What is the value of limited feedback for MIMO channels?", IEEE Communications Magazine, vol. 42, no. 10, pp.54-59, Oct. 2004.

[5] L. Hanzo, P. Cherriman and J. Streit, Video Compression and Communications: From Basics To H.261, H.263, H.264, MPEG4 For DVB and HSDPA-Style Adaptive Turbo-Transceivers. John Wiley and Sons Inc, 2007.

[6] K. Kabayashi, T. Ohtsuki and T. Kaneko, "MIMO systems in the presence of feedback delay", IEEE International Conference on Communications, vol. 9, pp. 41024106, Jun. 2006.

[7] R.L. Choi and R.D. Murch, "New transmit schemes and simplified receivers for MIMO wireless communication systems", IEEE Transactions on Wireless Communications, vol. 2, no. 6, pp. 1217-1230, Nov. 2003.

[8] M. Joham, W. Utschick, and J.A. Nossek, "Linear transmit processing in MIMO communications systems", IEEE Transactions on Signal Processing, vol. 53, no. 8, pp. 2700-2712, Aug. 2005.

[9] L.-L. Yang, "Design Linear Multiuser Transmitters from Linear Multiuser Receivers", IEEE International Conference on Communications, June 24th-28th, 2007.

[10] A. Gersho and R.M. Gray, Vector Quantization and Signal Compression. Boston/Dordrecht/London: Kluwer Academic Publishers, 1991.

[11] D.J. Love, R.W. Heath, Jr. and T. Strohmer, "Grassmannian Beamforming for Multiple-Input Multiple-Output Wireless Systems", IEEE Transcations on Information Theory Special Issue on MIMO communications, vol. 49, pp. 2735-2747, Oct. 2003.

[12] D. Yang, L.-L. Yang and L. Hanzo, "Performance of MIMO Systems Using Transmitter Preprocessing Based on Limited Noisy Feedback", IEEE 65th Conference on Vehicular Technology, pp. 2119-2123, April, 2007.

[13] TS 25.214 3GPP TSG RAN WG4, v.5.0.0, Physical Layer Procedures (FDD), 2002 03. 